\title{
DESAFÍOS ÉTICOS EN INVESTIGACIÓN GENÓMICA Y BIOTECNOLOGÍA. VEINTE AÑOS DE ACTA BIOETHICA
}

\begin{abstract}
Eduardo Rodríguez Yunta ${ }^{1}$
Resumen: El presente artículo es una revisión de las reflexiones éticas sobre temas de genética y manipulación genética que se han publicado en 20 años de Acta Bioethica. Se identifican los siguientes temas relevantes en el diálogo bioético: evaluación de riesgos, inequidad, pérdida de la biodiversidad, eugenesia, definir los límites entre natural y artificial, diálogo entre detractores y promotores de la biotecnología, desafíos en el cuidado de la salud, manejo de la información y confidencialidad. Se concluye que la bioética puede actuar como intermediaria entre los diversos interlocutores ante los problemas que se suscitan al aplicar la tecnología a la vida, permitiendo reconocer y llegar a consensos para enfrentar con responsabilidad las diferencias en temas de equidad, autonomía y el balance entre riesgos y beneficios. Las reflexiones en Acta Bioethica sobre genómica son similares a las de otras revistas, solo que el énfasis es en la región latinoamericana. En veinte años de reflexión, los desafíos siguen siendo los mismos, aunque el énfasis está cambiando de la prevención y rechazo en cuanto a los riesgos de la modificación genética, a la regulación de la tecnología de ingeniería genética, que va perfeccionándose cada vez más. En cuanto a los beneficios, la medicina genómica siempre ha generado más expectativas que realidades, sin lograr demostrar eficacia en ensayos clínicos. Con las nuevas técnicas de edición genómica, se amplían aún más las expectativas de mejores intervenciones, pero aumenta la preocupación de que se realicen modificaciones genéticas sin finalidad terapéutica, afectando al equilibrio social y ecológico.
\end{abstract}

Palabras clave: genética, biotecnología, bioética, modificaciones genéticas

Ethical challenges present in genomic research and biotechnology. Twenty years of Acta Bioethica

\begin{abstract}
This article reviews ethical reflections about genetic research and genetic manipulation published in 20 years of Acta Bioethica. The following relevant topics are identified in bioethics dialogue: risks evaluation, inequity, loss of biodiversity, eugenics, definition of limits between artificial and natural, dialogue between detractors and promoters of biotechnology, challenges in health care, information and confidentiality management. Reflections consider that bioethics can act as mediator among diverse stakeholders affronting the problems that arise when applying technology to life, allowing to recognize and to reach consensus for solving differences in equity, autonomy and the balance of risks and benefices. The reflections in Acta Bioethica are similar to those of other journals, but with emphasis in the Latin American region. In twenty years of reflection, the challenges are similar, but the emphasis is changing from preventing to the avoidance of actual risks of genetic modifications, which demands regulation of genetic engineering, taking into account that it is more effective than before. Among the benefits, genomic medicine has always generated more expectations than actual therapy with problems in demonstrating efficacy in clinical trials. With the new technique of genomic edition, there are even more expectations for enhancing therapeutic approaches, but worries increase about using genetic modifications without therapeutic aim and interventions that affect the social and ecological equilibrium.
\end{abstract}

Key words: genetics, biotechnology, bioethics, genetic modifications

\section{Desafios éticos na pesquisa genômica e biotecnológica. Vinte anos de Acta Bioethica}

Resumo: O presente artigo é uma revisão das reflexões éticas sobre temas de genética e manipulação genética que foram publicadas em 20 anos de Acta Bioethica. Os seguintes temas de importância para o diálogo bioético foram identificados: avaliaçáo de riscos, inequidade, perda da biodiversidade, eugenia, definir os limites entre natural e artificial, diálogo entre opositores e defensores da biotecnologia, desafios em cuidados à saúde, manejo da informaçáo e confidencialidade. Concluiu-se que a bioética pode atuar como intermediaria entre os diversos interlocutores ante os problemas que aparecem ao aplicar a tecnologia à vida, permitindo reconhecer e alcançar consensos para enfrentar com responsabilidade as diferenças em temas de equidade e autonomia, e o equilíbrio entre riscos e benefícios. As reflexóes na Acta Bioethica sobre genômica são similares às de outras revistas, mas com ênfase na região latino-americana. Em vinte anos de reflexão, os desafios seguem sendo os mesmos, ainda que a ênfase esteja mudando da prevenção e repúdio quanto aos riscos da modificação genética, à regulação da tecnologia de engenharia genética, que se aperfeiçoa cada vez mais. Quanto aos benefícios, a medicina genômica sempre gerou mais expectativas que realidades, sem conseguir demonstrar eficácia em ensaios clínicos. Com as novas técnicas de ediçâo genômica, ampliam-se ainda mais as expectativas de melhores intervençóes, mas aumenta a preocupação de que se realizem modificaçóes genéticas sem finalidade terapêutica, afetando o equilíbrio social e ecológico.

Palavras chave: genética, biotecnologia, bioética, modificaçóes genéticas

\footnotetext{
${ }^{1}$ Centro Interdisciplinario de Estudios en Bioética, Universidad de Chile, Chile. ORCID 0000-0002-7947-3868

Correspondencia: erodriguezchi@gmail.com
} 


\section{Introducción}

La manipulación genética consiste en la aplicación de técnicas dirigidas a modificar el caudal hereditario de alguna especie, con fines variables, desde la superación de enfermedades de origen genético (terapia genética) a la mejoría de características propias de la especie o con finalidad experimental o industrial (conseguir un individuo con características no existentes hasta ese momento). El hecho de poder modificar el acervo genético genera numerosas expectativas, como mejorar la producción de alimentos; en agricultura, mejorar la resistencia a plagas, enfermedades y adaptación a condiciones ambientales adversas; posibilidad de nuevas terapias y efectividad de vacunas; mejorar los trasplantes de órganos o poder usar fármacos individualizados sin efectos colaterales o con efectividad controlada. La farmacogenética permite identificar secuencias génicas y productos génicos asociados con enfermedades, que pueden actuar como señales para la actuación de fármacos y también identificar los perfiles genéticos asociados con efectos secundarios o adversos de la medicina(1). El descubrimiento de las variantes genéticas de los individuos que influyen en el efecto de los fármacos, permite el desarrollo de nuevos procedimientos diagnósticos y productos terapéuticos que se prescriben selectivamente a los pacientes, con garantías de seguridad y efectividad. También, el conocimiento sobre el genoma, tanto en el ser humano como en todo ser viviente, afecta la forma de plantearse el ser humano filosóficamente y a nivel de creencias en relación con la Naturaleza(2).

La posibilidad de generar modificaciones genéticas con aplicación industrial ha generado un gran interés en la comercialización de productos, protegiéndose las modificaciones con patentes, que otorgan el derecho exclusivo de comercialización por 20 ańos. Las empresas que tienen la tecnología se hallan en ventaja para la obtención de patentes, además de contar con los recursos para invertir en innovación. La biotecnología puede ser una fuente de desarrollo, pero muchos países se encuentran en desventaja. A pesar de ello, existen numerosas iniciativas para el desarrollo de capacidades de biotecnología en países en desarrollo(3).

Por otra parte, preocupa que, debido al uso de la biotecnología, sea posible la manipulación genética dirigida, entregando al ser humano un poder que puede afectar a los organismos vivos, alterándolos irreversiblemente(4).

\section{Seres vivos y tecnología}

El creciente proceso de globalización ha encontrado en la biotecnología un aliado para la transformación económica y social. Se considera que existe un sistema tecnológico siempre que alguien transforma intencionalmente un objeto concreto bajo un conjunto de creencias, conocimientos y valores(5). En la evaluación del sistema tecnológico y sus resultados pueden chocar sistemas de valores e intereses. Cuando el objeto es un ser vivo, hay que tener en cuenta que éste también posee intencionalidad y que puede ofrecer resistencia o quedar afectado por la transformación. El uso de la tecnología en seres vivos produce numerosos conflictos, al chocar creencias y valores entre diferentes grupos sociales, como los ecologistas, los científicos, los empresarios, agricultores, ganaderos, el ciudadano común e incluso el ser vivo objeto de transformación.

Se aduce la necesidad de que el Derecho, en relación al genoma humano y la biotecnología con seres vivos, evolucione desde normas jurídicas no obligatorias a normas con carácter obligatorio, respaldadas por sanciones con valores que deben respetarse, como la responsabilidad para con las futuras generaciones, la protección de la identidad del ser humano o el reconocimiento de la diversidad biológica(6).

\section{Problemas éticos por el uso de la modificación genética y la biotecnología}

\section{Evaluación de riesgos}

Desde el inicio de la biotecnología, ha habido preocupación por el uso de la tecnología del ADN recombinante en seres vivos y respecto de cómo medir el riesgo que implica. Ya en 1975, en la conferencia de Asilomar, hubo una moratoria, porque se consideraba peligroso trabajar con secuencias de ADN: había que hacerlo en lugares confinados por desconocimiento de su efecto en los sistemas ecológicos(7). 
El análisis de riesgo consta de tres elementos(8):

a) evaluación de riesgo, identificando los peligros potenciales y reales;

b) gestión de riesgo, estableciendo las medidas para minimizar o eliminar riesgos;

c) comunicación de riesgo hacia el público, especificando que proviene de un organismo genéticamente modificado.

Los organismos genéticamente modificados actúan con independencia del ser humano una vez generados, con propiedades nuevas no seleccionadas por la naturaleza, lo que puede provocar situaciones de nocividad ambiental, contaminación genética y alteración de ecosistemas(4).

La información y el conocimiento para la identificación, estimación y gestión de un riesgo, depende del contexto y de los fines, intereses y valores de quién los evalúa, por lo que constituye un desafío ético sujeto a diferencias entre los grupos sociales(5). La evaluación de riesgo no debe ser solo cuestión de expertos y no depende solamente de un cálculo de probabilidades por experiencias pasadas. La percepción y evaluación del riesgo se basa en un sistema de valores que varía en función de quienes hacen la evaluación(5).

\section{Inequidad}

La biotecnología está ahondando las diferencias entre países, entre los países y empresas productoras de tecnología, y al interior de los países que aportan la materia prima pero carecen de infraestructura tecnológica. Por lo general, el conocimiento tecnológico se desarrolla en los países ya desarrollados, el cual se encuentra altamente protegido y no existe una transferencia real(4). Esta asimetría se profundiza por el impacto económico que produce en las diferencias entre países. La biotecnología nació con la promesa de disminuir el hambre en el mundo, pero la verdadera causa del hambre es la desigualdad y situaciones de corrupción que no se resuelven con un aumento en la productividad(9). Por otra parte, la biotecnología trata a la naturaleza y los seres vivos como una mercancía y hace a agricultores y ganaderos dependientes de empresas(9). En agricultura, las empresas obligan a los agricultores a comprar semillas e insumos y les prohíben guardar, compartir o vender las semillas.

\section{Pérdida de biodiversidad}

La biotecnología utiliza como materia prima para su desarrollo a la biodiversidad genética(4). La biodiversidad se define como la variabilidad de organismos vivos de cualquier fuente, incluidos, entre otros, los ecosistemas terrestres y acuáticos, y los complejos ecológicos de los que forman parte; comprende la diversidad dentro de cada especie, entre las especies y de los ecosistemas(10). Es necesario asumir la biodiversidad como un sistema autorregulado, con el que el ser humano interactúa y del que es también parte(4). Es el ser humano el que puede afectar el equilibrio de este sistema en su mayor parte. Una de las mayores preocupaciones es la creciente desforestación por la demanda de productividad y de utilizar tierras dedicadas al cultivo para alimentar la humanidad. Por otra parte, Latinoamérica es la mayor región en biodiversidad del planeta (40\%), por lo que se necesita para ella mayor protección(11). Sin embargo, la riqueza en biodiversidad proporciona a la región pocos beneficios, los beneficios más bien caen en manos de empresas biotecnológicas y farmacológicas de capital extranjero, que patentan secuencias, seres vivos y fármacos, mientras que los países latinoamericanos actúan más bien como consumidores, siendo el número de patentes muy bajo(12). La tendencia de la biotecnología es formar mercados internacionales de semillas uniformes, afectando las posibilidades de conservación y mantenimiento de la diversidad genética a nivel local(9). Los cultivos transgénicos por lo general favorecen monocultivos caracterizados por la homogeneidad genética, lo cual les hace más vulnerables al estrés biótico y abiótico(13).

La diversidad genética, que antes era propiedad de nadie, ahora se ha privatizado por medio de la propiedad intelectual(4).

\section{Posibilidad de engenesia}

La eugenesia se refiere a mejorar las características humanas por selección de caracteres hereditarios considerados deseables, y la eliminación de los considerados indeseables. La idea de "eugenesia" ha estado presente desde la antigüedad. A lo largo de la historia se ha manifestado en mejorar la raza y eliminar enfermedades y genes defectuosos y características sociales indeseables. Bajo la ideología 
de la eugenesia subyace la idea de que es posible perfeccionar al ser humano, que existen seres humanos de menor categoría y que el progreso social se asocia a la perfección biológica y psicológica(14). Las ideas eugenésicas parten del ideal de perfeccionamiento del ser humano mediante la ingeniería genética como algo beneficioso para el ser humano, y la generación de una nueva humanidad o posthumanidad, pero se critica que no existe conexión entre mejorar las capacidades y el bienestar(15).

Hoy, la posibilidad de manipular el genoma abre la puerta a una nueva forma de eugenesia, al ser posible mejorar todo tipo de características, en forma idealista y de acuerdo con decisiones individuales por voluntad de consumidores de la tecnología. Por otra parte, existiendo presión social de eliminar a los fetos con anomalías $(16,17)$. Ante esta posibilidad, la pregunta es si resulta éticamente deseable perfeccionar el ser humano y cuáles son los límites. Además, conduce a la falta de protección del feto y del embrión. La selección de embriones en procedimientos de fecundación in vitro permite elegir el embrión con la información genética deseada por los progenitores, pero estas prácticas cuestionan la dignidad humana(18). La aceptación de la mejoría perfectiva en la sociedad, mediante manipulación genética, llevaría al aumento de diferencias sociales, a la discriminación y a la devaluación de ciertas categorías de personas, cuyos genes estarían asociados a problemas sociales(18). Se cuestiona el uso de la terapia génica germinal, por el problema de que no se puede obtener consentimiento de las generaciones futuras y, en el estado actual de la técnica, se violaría el principio de no maleficencia, por la posibilidad de riesgos de mutaciones que pasaran al acervo genético heredado $(18,19)$.

\section{Definir los limites entre artificial y natural}

Hasta el advenimiento de la ingeniería genética, no se disponía de una tecnología que permitiera rediseñar, de forma rápida y controlada, organismos adaptados. La biotecnología es capaz de superar las restricciones que existen entre especies, rompiendo límites que se suponían infranqueables, al operarse directamente en el genoma, por lo que se puede hablar de una "tecnificación del ser vivo". Al manipular el $\mathrm{ADN}$ de los seres vi- vientes, se puede trastornar su dimensión simbólica y ecológica ocupando nichos distintos(20). Se cuestiona que la modificación genética de organismos vivos trasforme su estatuto ontológico, pasando a ser un "organismo artificial”, capaz de reproducirse, pero instrumentalizado por el ser humano(21). Se desdibuja, por tanto, el límite entre lo natural y lo artificial.

\section{Diálogo entre detractores y promotores de la bio- tecnología}

Si bien, para algunos, la biotecnología abre numerosas posibilidades para el desarrollo de la humanidad, como la aparición de nuevas terapias, resolver el problema de la alimentación o salvar de la extinción a especies; para otros, la biotecnología representa la generación de numerosos males, como el desequilibrio ecológico, instrumentación del ser vivo o daños a la salud(22). El rechazo de los detractores de la manipulación genética en biotecnología se debe, en parte, a la falta de información por un buen sector de la sociedad y, por otra, a diferentes percepciones de riesgo. La comunicación de los avances en genética a la comunidad no especializada está cargada de intereses(23). La comunicación asociada a las empresas generalmente enfatiza los beneficios de los avances biotecnológicos, pero, por ejemplo, las promesas de la ingeniería genética para la agricultura no se han cumplido, como menor dependencia de insumos químicos, aumento de la productividad o disminución de costos de insumos, lo que ayudaría a reducir problemas ambientales(9). La mayor parte de las innovaciones en biotecnología de alimentos se orientan a ganancias para las empresas, más que a necesidades(9).

Aquellos que están interesados en la no intervención de la naturaleza enfatizan en sus comunicaciones los riesgos y peligros de la aplicación de la nueva biotecnología. La aplicación en agricultura y la producción de alimentos generan desconfianza pública en números contextos; así, por ejemplo, muchos consumidores tienen la percepción de que el hecho de que los alimentos hayan sido manipulados genéticamente los hace más peligrosos que los manipulados(24). En general, existe desconocimiento de los consumidores sobre el valor de la ingeniería genética para mejorar la calidad de alimentos(24). Existe también desinformación 
pública sobre los mecanismos de evaluación de riesgos utilizados por agencias de control(25). A menudo la biotecnología se presenta amenazante, pronosticando desenlaces fatales en forma irreversible, como la alteración de ecosistemas naturales al introducir especies transgénicas, mutaciones dañinas que podrían generarse en el laboratorio y efectos negativos en la salud de animales y seres humanos que consumen alimentos genéticamente modificados(26). El manejo sensacionalista de los medios produce un desajuste en relación con las medidas que podrían tomarse para mejoras en salud y desarrollo social, de acuerdo con bases científicas y participación ciudadana(27). Contar con la información adecuada, posibilitaría que los responsables de formular políticas pudieran asignar, de una manera racional, los escasos recursos disponibles en países en desarrollo, priorizando tecnologías y asegurando acceso equitativo, pero esto a menudo no ocurre(28).

Falta diálogo y encontrar un espacio de participación social para dilucidar las consecuencias de la biotecnología, y para prevenir, paliar o contrarrestar posibles efectos negativos(5). En las decisiones sobre el uso de organismos genéticamente modificados debieran participar todos los grupos afectados: científicos, tecnólogos, empresarios, ecologistas, comunicadores, políticos, bioeticistas, sociólogos, ciudadanos y trabajadores del campo afectado. Las conclusiones de cada grupo son necesariamente incompletas y ninguno debiera tener privilegio especial. Las diversas partes interesadas deben intercambiar información y proponer y debatir con razonamientos los métodos, aplicaciones y gestión de riesgos que se deben seguir(5).

\section{Desafíos en el cuidado de la salud}

Identificados los genes con sus funciones, a través del proyecto del genoma humano, e identificadas las numerosas mutaciones que producen enfermedades, surge la medicina genómica, con su potencial para prevención, diagnóstico y desarrollo de nuevas terapias, y el desarrollo de una medicina más personalizada, de acuerdo con el acervo genético del individuo; pero también surge la posibilidad de perfeccionar al ser humano o intervenir en la línea germinal. Se cuestiona la presentación exitista de la terapia génica, por la que se pueden corregir mutaciones mediante la manipulación genética, cuando todavía se está trabajando en la demostración de la eficacia clínica y no se transparenta del todo los riesgos asociados a la técnica(29). Los avances en medicina genómica generan el mito ideal de la salud perfecta que, en muchas ocasiones, ocasiona un alto costo en el cuidado de la salud y permite abusos sobre los derechos de las personas en sociedades poco democráticas, fundamentalmente porque no se respeta la autonomía de las personas y se da más énfasis a la comercialización que a ponerse al servicio del ser humano(30). La asesoría genética es un proceso de comunicación, que permite reconocer las características de las afecciones genéticas con su carga de enfermedad, los riesgos de ocurrencia y recurrencia, las posibilidades de terapia y los impactos en las familias(31). En la asesoría genética se pueden producir problemas por falta de comprensión, afectando a la autonomía, y por no proteger la confidencialidad y privacidad de las personas(32). Las explicaciones deben ser en términos muy simples, en forma imparcial y objetiva, apoyando y ayudando a la persona en su propia decisión.

En relación con la salud, el uso de pruebas genéticas para diagnóstico, prevención o terapia genera las siguientes inquietudes en Latinoamérica: falta de equidad en el acceso a los beneficios, ya que una muy pequeña fracción de la población tiene acceso; explotación comercial por empresas; posibilidad de estigmatización y discriminación genética; instrumentación de personas, quiebres de confidencialidad, usar las pruebas para diagnóstico y aborto eugenésico, o para selección de embriones; rechazo de las comunidades indígenas en investigación, por el inadecuado uso del consentimiento informado y la falta de regulaciones específicas en los países(33). En el debate se halla también la aceptación ética de la clonación humana con fines terapéuticas, siempre que sea para aliviar el sufrimiento humano $(34,35)$. Se crítica el reduccionismo y determinismo genético, debido a que se enfatiza exageradamente el papel de los genes en enfermedades y comportamientos(22). Se cuestiona que se quiera imponer el determinismo genético, sin tener en cuenta que los seres humanos también son producto de la interacción con el medio biológico y social, además de depender de su dotación genética(36). 
En relación con la farmacogenética, se cuestiona que se clasifiquen los sujetos por etnias, lo que puede llevar a estigmatización o discriminaciones. También se menciona el problema de la falta de equidad y que, si el ensayo clínico de un fármaco se efectuase en condiciones ideales respecto a toxicidad, con mínimo costo para la industria farmacéutica, y luego el fármaco estuviera disponible globalmente, incluyendo países donde el acceso a pruebas farmacogenéticas es muy restringido, provocaría que los eventos adversos se detectarían después de que el fármaco se comercializase y los daños los recibiría la población socioeconómica vulnerable(37).

\section{Manejo de la información y confidencialidad}

La información genética de los individuos puede constituirse en un instrumento de poder en manos de terceros, por lo que es crítico salvaguardar la confidencialidad y que se respete la voluntad de quien dona material genético(17). Se generan sistemas de información acerca de la genética humana, cuya expresión más consumada son los biobancos, en los que se almacenan muestras de tejidos de los que se puede extraer información genética y, con ella, realizar investigación. El empleo de las muestras almacenadas y de información asociada en los biobancos, genera múltiples expectativas para la investigación en salud y para realizar modificaciones genéticas. La administración de esta información implica riesgos que inciden en lo social, lo económico y lo político, como transgresiones a la dignidad, uso descuidado o uso militar o terrorista(38). Las excepciones de confidencialidad las determina la voluntad del donante, por consentimiento informado o un mandato judicial(39). La autonomía queda en riesgo cuando los sujetos se encuentran vulnerados socioculturalmente, como es el caso de genética forense de condenados(40).

En relación con las muestras de tejidos humanos de las que se extrae información genética, se cuestiona que pueda ser objeto de lucro comercial. La muestra biológica está vinculada al sujeto y se hace casi imposible la anonimización; en la justificación de patentes se aduce que, al ser una muestra modificada por el investigador, está ya no es propiedad del donante, pero en realidad la muestra sigue manteniendo un vínculo con el donante, por muchas transformaciones que se realicen(1). Dado el enorme avance de la biotecnología, se necesita reconocer la variedad de formas de crear vida y de generar contenidos vitales a partir de muestras de origen humano, lo que exige ser sometido a regulación; la idea de ser sujeto de derecho debe enmarcarse dentro de ésta regulación(41). Para otros, antes de proteger las muestras biológicas y datos asociados de posibles intrusiones, es necesario diseñar estrategias para promover el acceso justo y equitativo(42).

Se cuestiona que, al ser el material genético compartido en parte en las etnias, la autonomía de un individuo, por participar en un estudio de investigación, puede chocar con la autonomía de otros individuos de la etnia que no participan, ya que las consecuencias de los estudios genéticos pueden afectar a un grupo entero(1). Preocupa también la falta de reconocimiento de los conocimientos tradicionales indígenas - o etnoconocimientosobre propiedades terapéuticas en plantas, y el aprovechamiento de la industria al usar este conocimiento sin reconocer su origen ni compensar a la población indígena por su obtención(12). Los pueblos indígenas cuestionan los derechos de propiedad intelectual, ya que éstos comercializan conocimientos que son tradicionales, originados en las etnias, y lo hacen en términos no equitativos. Además, en la cultura indígena no tiene cabida el derecho de propiedad si no es en forma colectiva $\mathrm{y}$, aunque los derechos indígenas hayan sido reconocidos en la ONU, no ha habido aceptación en los Estados, debido a intereses de empresas, por lo que no se reconocen los recursos genéticos como propiedad cultural de etnias(43).

\section{Rol de la bioética}

La práctica de la bioética ha permitido desarrollar herramientas de diálogo y deliberación, en forma interdisciplinaria, entre legos y expertos. La deliberación ética, con el uso del diálogo y la argumentación, de manera que todas las partes sean escuchadas para encontrar consensos, es fundamental para resolver problemas éticos(44). La comunicación bioética se constituye como un medio de contención, que relaciona el ámbito de la investigación biológica con otros sistemas sociales, dando forma a la diferencia entre el poder hacer de la biotecnología y el deber hacer por res- 
ponsabilidad social(26). La deliberación en bioética puede actuar como intermediaria entre los diversos interlocutores, ante los problemas que se suscitan al aplicar la tecnología a la vida, permitiendo reconocer y llegar a consensos para enfrentar con responsabilidad las diferencias en temas de equidad, autonomía y el balance entre riesgos y beneficios. La bioética puede actuar como intermediario entre la antropología social, la ética, la ciencia y la tecnología, señalando límites en la aplicación de estas últimas, para que su poder no resulte en trasgresiones. La reflexión bioética puede ayudar a que los proyectos biotecnológicos sean sometidos a regulación social, ponderando beneficios y riesgos y promoviendo la equidad en el acceso a los bienes producidos(45).

Una de las dificultades es encontrar mecanismos de participación ciudadana. Se propone realizar "coloquios de consenso" — talleres de discusiónque permitan a grupos de ciudadanos participar en la evaluación de sistemas tecnológicos en conjunto con expertos, medios de comunicación, responsables de la elaboración de políticas y representantes de empresarios(5).

De relevancia es el principio de responsabilidad, postulado por Han Jonas para la era de la biotecnología como una forma de imputar las acciones del pasado, y que se orienta al futuro más allá de las consecuencias que se puedan prever y en el que la sociedad en su conjunto debe tomar parte(46).
Los avances en tecnología genética han posibilitado la edición genómica o modificación del ADN en forma dirigida, lo que abre la puerta al mejoramiento de la especie humana y a intervenir la línea germinal, siempre que se superen los riesgos técnicos. Debido a estos avances, se ve la necesidad tanto de un análisis bioético como biojurídico en cuanto a cómo limitar la técnica. El dilema ya no es solamente ético individual, sino que afecta a la sociedad y la condición humana(47).

En veinte años de reflexión sobre la ética de investigación genómica, los desafíos siguen siendo los mismos, aunque el énfasis está cambiando de la prevención y rechazo en cuanto a los riesgos de la modificación genética, a la regulación de la tecnología de ingeniería genética que va perfeccionándose cada vez más. En cuanto a los beneficios, la medicina genómica siempre ha generado más expectativas que realidades, sin lograr demostrar eficacia en ensayos clínicos. Con las nuevas técnicas de edición genómica, se amplían aún más las expectativas de mejores intervenciones, pero aumenta la preocupación de que se realicen modificaciones genéticas afectando al equilibrio social y ecológico. Se cuestiona, por ejemplo:

Las estructuras actuales, ¿'son suficientes para evaluar riesgos sociales a largo plazo?

¿Cómo impactan las creencias religiosas y las tradiciones culturales en el uso de edición genómica para la aceptabilidad social?

$\mathrm{Al}$ introducir la tecnología de edición genómica, ¿cómo abordar los vacíos en regulación, los procedimientos de evaluación ética y la consideración de conflictos de interés?

¿Cuál sería una autorización justa y legítima para ensayos de liberación de organismos editados, cuando puede impactar el equilibrio ecológico? 
Desafíos éticos en investigación genómica y biotecnología. Veinte años de Acta Bioethica - Eduardo Rodríguez Yunta

\section{Referencias}

1. Bota Arqué A. El reto de la muestra biológica en los estudios farmacogenéticos. Acta Bioethica 2004; 10(2): 201-212. DOI: https://dx.doi.org/10.4067/S1726-569X2004000200008

2. Santos M, Vargas L. Valuación bioética del proyecto genoma humano. Acta Bioethica 2002; 8(1): 111-123. DOI: https://dx.doi.org/10.4067/S1726-569X2002000100011.

3. Daar A, Sahni P, Singer P. Genomics, biotechnology and global health: the work of the University of Toronto joint centre for bioethics. Acta Bioethica 2004; 10(2): 213-225. Disponible en https://actabioethica.uchile.cl/index.php/AB/ article/view/16763/17462.

4. Bota Arqué A. El impacto de la biotecnología en América Latina: espacios de participación social. Acta Bioethica 2003; 9(1): 1-18. DOI: https://dx.doi.org/10.4067/S1726-569X2003000100003.

5. Olivé L. Los objetos biotecnológicos: concepciones filosóficas y consecuencias para su evaluación. Acta Bioethica 2003 ; 9(1): 9-19. Disponible en https://scielo.conicyt.cl/scielo.php?script=sci_arttext\&pid=S1726569X2003000100002\&lng=en. http://dx.doi.org/10.4067/S1726-569X2003000100002.

6. Romeo Casabona CM. La genética y la biotecnología en las fronteras del derecho. Acta Bioethica 2002; 8(2): 283297. DOI: https://dx.doi.org/10.4067/S1726-569X2002000200009.

7. Norman C. Genetic manipulation guidelines. Nature 1976; 262: 2-4.

8. Tzotzos, GT. Genetically modified organisms: a guide to biosafety. United Nations Industrial Development Organization; International Centre for Gewnetic Engineering and Biotechnology; UNIDO/UNEP/WHO/FAO Informal Working Group on Biosafety. Wallingford, Oxon, UK: CAB International; 1995.

9. Altieri MA. Dimensiones éticas de la crítica agroecológica a la biotecnología agrícola. Acta Bioethica 2003; 9(1): $47-61$. DOI: https://dx.doi.org/10.4067/S1726-569X2003000100005.

10. Conferencia de las Naciones Unidas sobre Medio Ambiente y Desarrollo. Convenio sobre Conservación y Uso Sostenible de la Diversidad Biológica, 1992.

11. Groombridge B, Jenkins MD. World Atlas of Biodiversity: Earth's Living Resources in the 21st Century. California: Ed. University of California; 2002.

12. Herrera Vásquez S, Rodríguez Yunta E. Etnoconocimiento en Latinoamérica. Apropiación de recursos genéticos y bioética. Acta Bioethica 2004; 10(2): 181-190. Disponible en https://actabioethica.uchile.cl/index.php/AB/article/ view/16759/17457

13. Robinson RA. Return to resistance: Breeding crops to reduce pesticide resistance. Davis: AgAceess; 1996.

14. Villela Cortés F, Linares Salgado JE. Eugenesia: Un análisis histórico y una posible propuesta. Acta Bioethica 2011; 17(2): 189-197. DOI: https://dx.doi.org/10.4067/S1726-569X2011000200005.

15. Vaccari A. La posthumanidad como un bien objetivo: los peligros del futurismo en el debate sobre la optimización genética humana. Acta Bioethica 2014; 20(2): 237-245. Disponible en https://revistaurbanismo.uchile.cl/index.php/ $\mathrm{AB} /$ article/view/33340/35064.

16. Antoine J-L. Genome and bioethics: an hollistic vision of the way we march towards the happy world biosciences promise. Acta Bioethica 2004; 10(2): 131-141. Disponible en https://revfono.uchile.cl/index.php/AB/article/view/16755/17452.

17. Bergel, S. Human rights: in between bioethics and genetics. Acta Bioethica 2002; 8(2): 315-331. Disponible en https:// actabioethica.uchile.cl/index.php/AB/article/view/16848/17548.

18. Alvarado MJ, Santos Alcántara M. Ethical Problems with the Preimplantation Genetic Diagnosis of Human Embryos. Acta Bioethica 2018; 24(1) 75-83. Disponible en https://actabioethica.uchile.cl/index.php/AB/article/ view/49379/57557.

19. Kohn Loncarica A, Outomuro D, Bortz J, Sánchez N. Gene therapy: medical treatment, eugenics or inheritanceôs hygiene? Acta Bioethica 2004; 10(2): 143-153. Disponible en https://revfono.uchile.cl/index.php/AB/article/ view/16756/17453.

20. Bota Arqué A. Editorial Biotecnología y Ética. Acta Bioethica 2003; 9(1).

21. Bota Arqué A. Animales transgénicos como organismos artificiales. Acta Bioethica 2007; 13(1): 61-70. DOI: https:// dx.doi.org/10.4067/S1726-569X2007000100007.

22. Mainetti JA. Bioética y genómica. Acta Bioethica 2003; 9(1): 39-46. Disponible en https://dx.doi.org/10.4067/S1726 569X2003000100004

23. Outomuro D, Mirabile LM, Cosmai N. El posicionamiento de los medios de comunicación entre la comunidad médica especializada en estudios genéticos. Acta Bioethica 2007; 13(2): 246-253. DOI: https://dx.doi.org/10.4067/S1726569X2007000200013. 
24. Correa Soares BE. Ethical points of view of biotechnology's public perception. Acta Bioethica 2003; 9(1): 63-67. Disponible en https://actabioethica.uchile.cl/index.php/AB/article/view/16817/17513.

25. Oda LM, Correa Soares BE. Genetically modified foods: economic aspects and public acceptance in Brazil. Trends in Biotechnology 2000; 18(5): 188-190.

26. Arnold Cathalifaud M. La comunicación genética desde la perspectiva de los sistemas sociales. Acta Bioethica 2002; 8(1): 67-86. DOI: https://dx.doi.org/10.4067/S1726-569X2002000100008.

27. Prieto González EA. Deterioro genómico y manipulación genética: desequilibrio en la prioridad de las agendas públicas. Acta Bioethica 2007; 13(2): 223-231. DOI: https://dx.doi.org/10.4067/S1726-569X2007000200010.

28. Martin C, Williams-Jones B, De Ortuzar MG. Ethical health technology assessment in Latin America: lessons from Canada and Argentina. Acta Bioethica 2011; 17(2): 225-236. DOI: https://dx.doi.org/10.4067/S1726569X2011000200009.

29. Rodríguez Yunta E. Gen therapy and ethical principles. Acta Bioethica 2003; 9(1): 69-79. Disponible en https://actabioethica.uchile.cl/index.php/AB/article/view/16819/17516

30. Vergès C. Genetics and bioethics in Latin America. Acta Bioethica 2004; 10(2): 155-166. Disponible en https://actabioethica.uchile.cl/index.php/AB/article/view/16757/17454.

31. Santos Alcántara MJ. Aspectos bioéticos del consejo genético en la era del proyecto del genoma humano. Acta Bioethica 2004; 10(2): 191-200. DOI: https://dx.doi.org/10.4067/S1726-569X2004000200007.

32. Gajardo Ugás A. El consejo genético desde una perspectiva bioética personalista. Acta Bioethica 2007; 13(2): 176180. DOI: https://dx.doi.org/10.4067/S1726-569X2007000200004.

33. Lolas Stepke F, Rodríguez Yunta E, Valdebenito Herrera C. El proyecto del genoma humano en la literatura biomédica en cuatro países latinoamericanos. Acta Bioethica 2004; 10(2): 167-180. Disponible en https://actabioethica.uchile.cl/ index.php/AB/article/view/16758/17455.

34. Arranz J, Ariza X, Riudor E, Vilar L, Salvadó J, Galofré N, Vendrell S, Esquirol J. Preliminar reflections about a present techno medical application: clonation. Acta Bioethica 2003; 9(1): 81-91. Disponible en https://actabioethica.uchile.cl/ index.php/AB/article/view/16822/17518.

35. Schramm RF. Promising health perspectives for human cloning. Acta Bioethica 2003; 9(1): 93-104. Disponible en https://actabioethica.uchile.cl/index.php/AB/article/view/16823/17519.

36. Sánchez González SR. Proyecto genoma humano visto desde el pensamiento de la complejidad: implicaciones bioéticas. Acta Bioethica 2008; 14(2): 142-147. DOI: https://dx.doi.org/10.4067/S1726-569X2008000200003.

37. Rodríguez E. Ética en innovación tecnológica y farmacogenómica. Acta Bioethica 2009; Monografía 2: $265-282$.

38. Villarroel R. Administración biopolítica de la intimidad en los biobancos. Acta Bioethica 2013; 19(1), 39-47. DOI: https://dx.doi.org/10.4067/S1726-569X2013000100005.

39. Sánchez Urrutia A. Genetic information, intimacy and discrimination. Acta Bioethica 2002; 8(2): 255-262. Disponible en https://actabioethica.uchile.cl/index.php/AB/article/view/16843/17542.

40. Grazinoli Garrido R, Garrido F. de S. R. G. Consentimiento informado en genética forense. Acta Bioethica 2013; 19(2): 299-306. DOI: https://dx.doi.org/10.4067/S1726-569X2013000200015.

41. Varsi Rospigliosi E. Clasificación del sujeto de derecho frente al avance de la genómica y la procreática. Acta Bioethica 2017; 23(2): 213-225. DOI: https://dx.doi.org/10.4067/S1726-569X2017000200213.

42. Siqueiros-García J, Oliva-Sánchez P, Saruwatari-Zavala G. Genomic sovereignty or the enemy. Acta Bioethica 2013; 19(2): 269-273. Disponible en https://revistaei.uchile.cl/index.php/AB/article/view/30006/31776.

43. Millaleo-Hernández S. Recursos genéticos y pueblos indígenas: la tesis de la propiedad cultural indígena frente al dominio público. Acta Bioethica 2019; 25(1): 51-61. Disponible en https://actabioethica.uchile.cl/index.php/AB/article/ view/53568/57127.

44. Gracia D. La confidencialidad de los datos genéticos. En: Gracia D. Ética de los Confines de la Vida. Bogotá: Editorial El Búho; 1998.

45. Schramm RF, Kottow Lang M. Bioética y biotecnología: lo humano entre dos paradigmas. Acta Bioethica 2001; 7(2): 259-267. DOI: https://dx.doi.org/10.4067/S1726-569X2001000200007.

46. Jonas H. Técnica, medicina y ética. La práctica del principio de responsabilidad. Barcelona: Paidós, 1997.

47. Cárdenas Krenz R. El Derecho ante la técnica de edición genética CRISPR. Acta Bioethica 2019; 25(2): 187-197. Disponible en https://actabioethica.uchile.cl/index.php/AB/article/view/54799/57876. 